\title{
Gold Nanorods as Photoacoustic Nanoprobes to Detect Pro-inflammatory Macrophages and Inflammation
}

Kabir S. Dhada, Derek S. Hernandez, Wenbai Huang, Laura J. Suggs

Department of Biomedical Engineering, University of Texas at Austin, Austin, Texas 78712

Corresponding Author: Laura J. Suggs, suggs@utexas.edu

\section{SUPPLEMENTARY FIGURES}

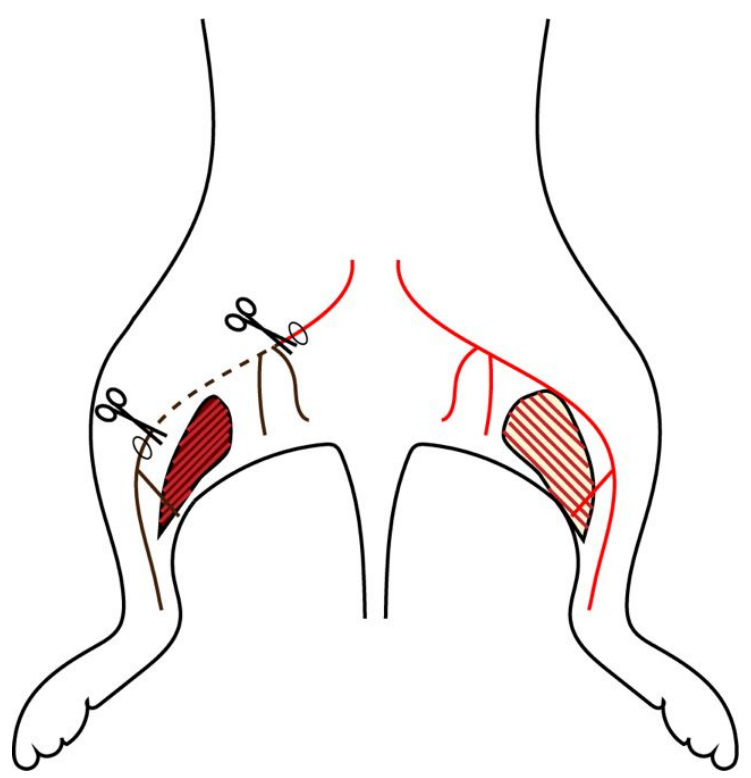

Supplementary Figure S1. Schematic of ligation procedure. Circles are suture points and the dotted line represents the portion of the artery that is resected. 

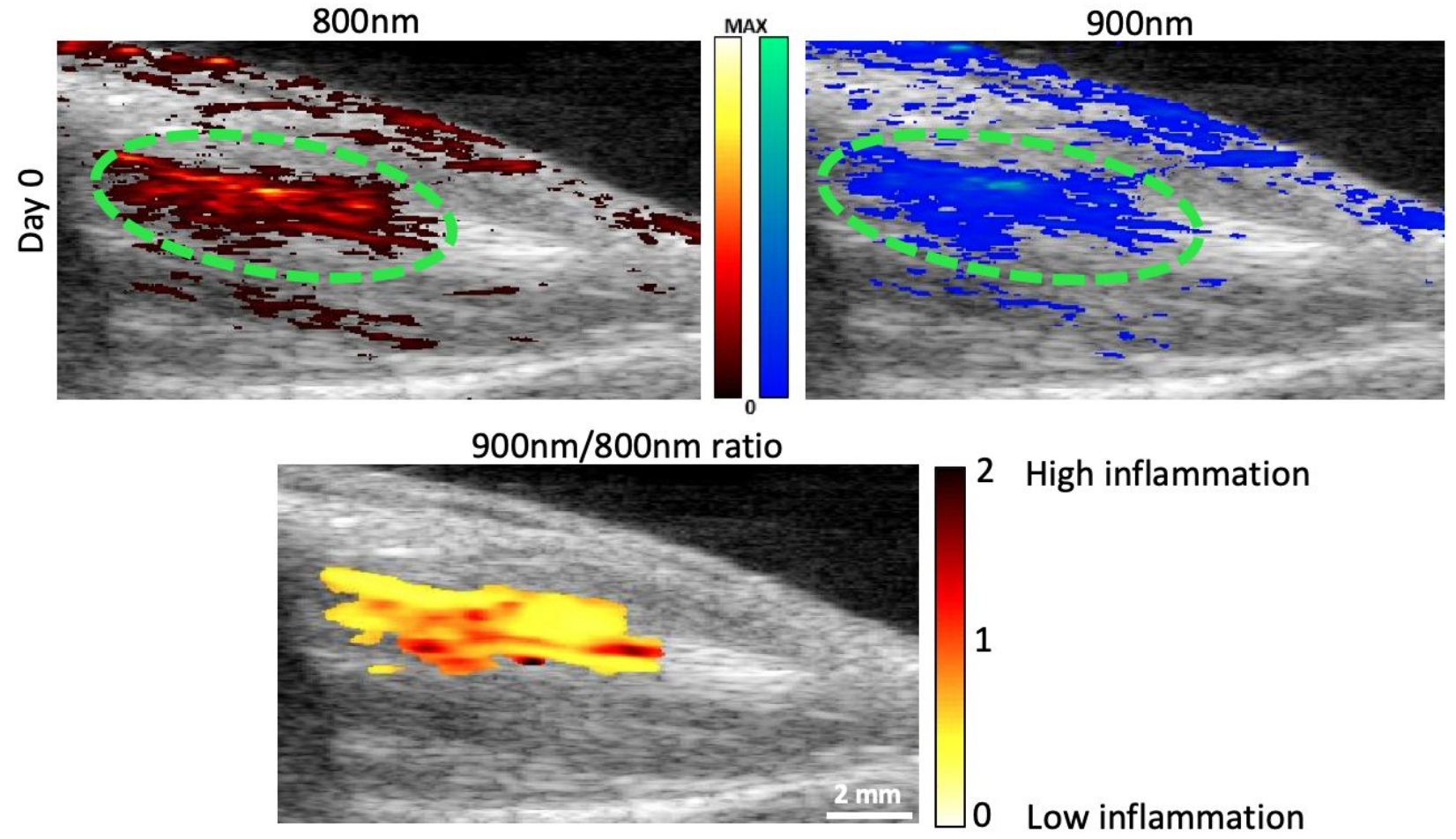

Supplementary Figure S2. Demonstration of ratiometric analysis used for inflammation grading. 